\title{
AVALIAÇÃO DO SISTEMA QUANTITATTVE BUFFY COAT (QBC) NO DIAGNÓSTICO LABORATORIAL DA INFECÇÃO PELO TRYPANOSOMA CRUZI: ESTUDO EM MODELO EXPERIMENTAL MURINO
}

\author{
Vicente Amato Neto, Luís Matsubara e Perla Natalia Bustos Lanura
}

Para esclarecer o diagnóstico da doença de Chagas e imprescindivel a confirmaça etiológica, dependente da utilização de diversos tipos de procedimentos, que demonstram o Trypanosoma cruzi ou participam revelando a natureza do processo através de anticorpos, por exemplo ${ }^{1+}$

A fase aguda pode apresentar-se através de manifestaçoes clínicas sugestivas, mas é definida segundo critério lahoratorial, ou seja, a evidenciaçào do $T$. cruzi no sangue periférico por meio de métodos rotulados como diretos e representados por exames a fresco ou após coloração, com concentração ou não. Contudo, torna-se necessário lembrar que ainda são desejáveis aprimoramentos, sobretudo quando vigentes pequenas parasitemias. Ainda mais, até em estudos experimentais, baseados no emprego de modelos que se utilizam de animais, vale a pena almejar progressos, para melhorar avaliaçòes patogenéticas e terapêuticas entre outras.

Quando tomamos conhecimento do valor do sistema Quantitative Buffy Coat (QBC) no diagnóstico específico da malária, em virtude da eficiência para mostrar hematozoários no sangue, julgamos aconselhável tentar verificar a aplicabilidade dessa técnica em relaçào ao $T$. cruzi e, de início, realizamos observaçoes recorrendo a camundongos intencionalmente infectados. Agora, então, relatamos os resultados obtidos.

Material e Métodos. Cinco camundongos fêmeas, da linhagem Balb-C, pesando entre 18

\footnotetext{
Laboratório de Investigação Médica - Parasitologia do Hospital das Clinicas da Faculdade de Medicina da Universidade de São Paulo, São Paulo, SP, Brasil.

Enderéço para correspondência: Prof. Vicente Amato Neto. Laboratório de Investigação Médica - Parasitologia. Av. Dr. Enéas de Carvalho Aguiar 500, 05403-000 São Paulo, SP
}

Recebido para publicação em 20/02/95 e $10 \mathrm{~g}$ cada um, forma inoculados pela via intraperitoneal com $10^{5}$ formas tripomastigotas da cepa Y do T. cruzi, mantida há muitos anos no Laboratório. Decorridos sete dias, efetuamos punçào cardíaca total, usamos citrato de sódio a $3,5 \%$ como anticoagulante e agrupamos em um único "pool", no qual contamos os flagelados empregando a câmara de Neubauer. Pusemos o sangue assim coletado em dez tubos capilares contendo laranja de acridina, atingindo o volume médio de 60 microlitros; homogeneizamos, colocamos rolha de silicone en uma das extremidades e bastonete de plástico, denominado flutuador, na outra; centrifugamos a $12.000 \mathrm{rpm}$ durante cinco minutos, para subseqüêntemente observar sob luz ultra-violeta, em microscópio de fluorescência, com objetiva de $60 \mathrm{X}$ e grande profundidade de campo; contamos e expressamos os resultados segundo o número médio concernente a dez capilares.

Para analisar a sensibilidade da técnica, juntamos sangue do "pool" ao de camundongos não infectados, a fim de conseguir concentrações de $1 / 20$ a $1 / 10$ 240. De cada uma delas pusemos amostra em um tubo capilar e contamos.

Comentários. Nos capilares, notamos nítida separação em diferentes camadas: plasma, plaquetas, mononucleados, granulócitos e hemácias. Aparece coloraçào esverdeada, fluorescente, nos núcleos, facilitando a indentificaçào. Quanto as formas tripomastigotas, elas são vistas apresentando grande motilidade e intensa fluorescência em cinetoplastos e núcleos, que é mais discreta nos citoplasmas.

A distribuição dos parasitas nos tubos pode ser expressa como está a seguir especificada: $75,9 \%$ no plasma, em região próxima às plaquetas; $15 \%$ na porção mais interna correspondente às plaquetas; $9 \%$ na camada de mononucleados e granulócitos (Tabela 1). 
Comunicação. Amato Neto V, Matsubara L, Lanura PNB. Avaliação do sistema quantitative buffy coat (QBC) no diagnóstico laboratorial da infeccâo pelo Trypanosoma cruzi: estudo em modelo experimental murino. Revista da Sociedade Brasileira de Medicina Tropical 29:59-61, jan-fev, 1996.

Tabela 1 - Nümeros de triponastigotas, à anâlise pelo sistema QBC, conforme diferentes camadas do sangue.

\begin{tabular}{lccc}
\hline Plasma & Plaquetas & $\begin{array}{c}\text { Mononucleados e } \\
\text { granulócitos }\end{array}$ \\
\hline & 846 & 101 & 84 \\
708 & 117 & 89 \\
& 589 & 172 & 74 \\
753 & 202 & 65 \\
& 834 & 158 & 99 \\
& 567 & 114 & 86 \\
& 786 & 147 & 87 \\
& 810 & 114 & 103 \\
Médias & 661 & 145 & 92 \\
\hline Porcentagcins & 823 & 193 & 96 \\
\hline
\end{tabular}

Passadas 24 horas, notamos redução, da ordem de $90 \%$, de flagelados vivos, estando os imóveis na parte superior do contingente de plaquetas, tendo morfologia preservada, cinetoplastos e núcleos visíveis com fluorescência e citoplasmas avermelhados, não fluorescentes.

A respeito das diluições, a partir de sangue com 200 tripomastigotas em $1 \mathrm{~mm}^{3}$, encontramos formas na totalidade e, até naquela de $1 / 10240$, existia uma única (Tabela 2).

Tabela 2 - Ntimeros de tripomastigotas, à análise pelo ststema $\mathrm{QBC}$ comforme diferentes dilucioses do sangue.

\begin{tabular}{cc} 
Conforme diferentes dilucoes do sangue. \\
\hline $1 / 20$ & 200 \\
$1 / 40$ & 180 \\
$1 / 80$ & 153 \\
$1 / 160$ & 102 \\
$1 / 320$ & 87 \\
$1 / 640$ & 54 \\
$1 / 1280$ & 18 \\
$1 / 2560$ & 32 \\
$1 / 5120$ & 7 \\
$1 / 10240$ & 1 \\
\hline
\end{tabular}

O sistema QBC é procedimento que promove concentração baseada no gradiente de densidade de diferentes componentes do sangue. Os constituintes do "creme" leucocitário, representados por plaquetas, mononucleados e granulócitos, passam a ocupar posição próxima à parede do capilar, ficando mais dispersos, de molde a facilitar a observação. A identificação de elementos nucleados, quer sejam parasitas ou não, tornase bastante simplificada como decorrência da afinidade que o corante tem com os ácidos nucléicos, advindo disso coloração esverdeada fluorescente mediante exame com o auxílio da luz ultravioleta.
Essa técnica tem se mostrado valiosa no diagnóstico da malária, mormente quando a parasitemia sanguínea é pequena. Essa circunstância é benéfica, sem dúvida, se bem que a ela contrapõem-se alguns fatos que devem ser levados em conta: dificuldade para separar Plasmodium falciparum de $P$. vivax; custo mais elevado do que o de processos habituais; pouca disponibilidade em certos lugares de países subdesenvolvidos. De qualquer maneira, possui maior sensibilidade do que outras técnicas, como a tradicional gota espessa.

No que concerne às tripanossomoses, vale registrar que o sistema QBC propiciou positividade nos sangues de ratos infectados pelo Trypanosoma brucei rhodesiense, mesmo quando extremamente pequenas as parasitemias ${ }^{3}$.

Podemos afirmar que a tentativa que empreendemos, para avaliar a efetividade do sistema com a finalidade de demonstrar o $T$. cruzi no sangue, conduziu a resultados animadores. Nâo houve interferência nas formas tripomastigotas, que mantiveram clara movimentação. A intensa fluorescência nas estruturas com ácidos nucléicos facilita a identificação, inclusive na vigência de imobilidade. Ocorreu marcante concentração de flagelados na faixa entre as plaquetas e o plasma, suscitando ainda ênfase à sensibilidade, pois na diluição a $1 / 10240$ estava um tripanossomo e, portanto, em 60 microlitros.

Facilidade à execução, visualização sem dificuldade e eficiência perante baixas parasitemias credenciam o método para utilização em trabalhos diagnósticos, terapêuticos e científicos. Já temos acumulada alguma experiência no que tange a acometimentos humanos, agudos, e em reativaçôes relacionadas com imunodeprimidos, derivando daí opinião satisfatória. Oportunamente, relataremos o que está sendo registrado. Outrossim, estamos analisando o sangue de infectados e em fase crônica; talvez a sensibilidade mude conhecimento consolidado, de acordo com o qual processos diretos não mostram o T. cruzi nessa etapa da parasitose.

\section{REFERÊNCIAS BIBLIOGRÁFICAS}

1. Camargo ME. Apreciação das técnicas sorológicas atuais para o diagnóstico da doença de Chagas. 
Comunicação. Amato Neto V, Matsubara L, Lanura PNB. Avaliação do sistema quantitative buffy coat (QBC) $n o$ diagnóstico laboratorial da infeccão pelo Trypanosoma cruzi: estudo em modelo experimental murino. Revista da Sociedade Brasileira de Medicina Tropical 29:59-61, jan-fev, 1996.

Revista da Sociedade Brasileira de Medicina Tropical 25(supl III): 10, 1992.

2. Garin B, Salun JJ, Peyron F, Vigier JP, Busangu I, Perrone J. Rapid in vivo detection of chloroquine resistance by the quantitative buffy coat malaria diagnosis system. The American Journal of Tropical Medicine and Hygiene 47:446-449, 1992.
3. Levine RA, Wardlaw SC, Patton CL. Detection of haematoparasites using quantitative buffy coat analysis tubes. Parasitology Today 5:132-134, 1989.

4. Luquetti AO. Diangóstico laboratorial da doença de Chagas. Revista da Sociedade Brasileira de Medicina Tropical 25(supl III):22-23, 1992. 